\title{
EXPERIMENTAL RESEARCH OF THE CLOSED ORBIT EXPANSION EFFECT IN THE STRONG FOCUSING
} CYCLOTRON

A.T.Vasilenko, A.A.Glazov, V.P.Dzhelepov, V.P.Dmitrievsky, Yu.N.Denisov,B.I.Zamolodchikov, N.I.Zaplatin, V.V.Kalinichenko, V.V.Kolga, D.L.Novikov, L.M.onischenko, N.I.Polumordvinova, E.V.Samsonov, P.T.Shishlyannikov

Laboratory of Nuclear Problems

Joint Institute for Nuclear Research, Dubna, USSR

\section{Summary}

The theoretical and experimental study of beam extraction method using the closed orbit expansion effect is described. The steep slope of the magnetic field variation magnitude is used to change the momentum compaction factor for the limit radial area. The orbit separation is found by computing the dynamical equations, since the behaviour of the betatron oscillation frequencies and the beam phase are investigated in this radial region. The experimental study of the effect is carried out with the ring cyclotron electron model, which is a strong focusing eight sector isochronous cyclotron. Calculated orbit separation in the extraction area is about $2 \div 4 \mathrm{~cm}$. The magnet system which is to obtain the proper gradient of the magnetic field variation and the magnet measurements results are described. The measured value of the orbit separation equals $4 \mathrm{~cm}$. At the same time the space between two orbits without current (free of particles) is about $2 \mathrm{~cm}$ and the full current on the separated orbit is equal to that before separation.

The theoretically predicted beam phase shift is found to be equal to $30+40^{\circ}$.

The obtained results confirm the possibility of the $100 \%$ beam extraction from the accelerator with space magnetic field variation.

\section{Introduction}

The basic problem in the design and construction of cyclic accelerator is a $100 \%$ beam extraction efficiency from the accelerator chamber. This problem is important for the meson facilities in the $1 \mathrm{GeV}$ energy range and the $1 \mathrm{~mA}$ current range due to the accelerated beam power which exceeds $1 \mathrm{MW}$ in this case.

The theoretical consideration of this problem has shown that all the methods of beam extraction based on free oscillation excitation cannot provide the full orbit separation. Thus, beam losses are inevitable due to finite barrier thickness.

The necessity of solving this problem made us search for methods neglecting the conventional excitation of free oscillations as well as the achievement of full separation emittance at the full radius without the noticeable emittance distortion. It has been established that the method satisfying these conditions is based on closed orbit expansion at full acceleration radii 2 .

The results of experimental and theoretical studies of this effect obtained by means of the electron model of the JINR strong focusing cyclotron are the subject of our paper 3 .

\section{Theoretical Basis and the Dynamical Calculations}

The magnetic field variation ( 6 ) results in the change of the mean radius $R$ of the closed orbit for the particle momentum $D$. which is characterized by the parameter $\lambda$

The mean radius $R$ can be found from the expression

$$
P=e B(R) R
$$

where $B(R)$ is the mean value of the magnetic field induction with the radius $R$, while

$$
\begin{array}{r}
\lambda=\frac{1}{2}+\sqrt{\frac{1}{4}+\frac{\epsilon^{2}}{2 N^{2}}\left\{\frac{3}{2}+n+\frac{R}{\epsilon} \frac{d \epsilon}{d R}\right)} \\
n=\frac{\mathbf{R d B}}{B d R} \quad, \epsilon=\frac{B N}{B(R)},
\end{array}
$$

$N$ is the periodicity of the magnetic field structure. The coefficient of closed orbit expansion is obtained directly from (1)

$$
\eta=\frac{p}{L} \frac{d L}{d p}=\frac{1}{1+n+\frac{A}{\lambda} \frac{d \lambda}{d R}} .
$$

The radial step of the closed orbit with the energy gain $\Delta E$ is, respectively,

$$
\frac{\Delta \mathbf{R}}{\mathbf{R}}=\eta \frac{\Delta \mathrm{p}}{\mathbf{p}}=\eta \frac{\Delta \mathbf{E}}{\beta^{2} \mathbf{E}} \text {. }
$$

As follows from (3) it is possible to control the coefficient of the closed orbit expansion by changing magnetic field variation The condition

$$
\mathbf{l}+\mathbf{n}+\frac{\mathbf{R}}{\lambda} \frac{\mathrm{d} \lambda}{d \mathbf{H}}=\mathbf{0}
$$

determines the sign of $\eta$ dividing the magnet structure into two classes: one having positive and the other one having negative $\eta$. A typical plot of expansion coefficient dependence obtained with the characteristics of the electron model magnetic field with a proper variation law is shown in Fig. 1 .

The magnetic system rigidity in the transverse direction to the closed orbit changes in the direction of the coefficient variation. However, the expansion itself occurs during one or two last revolutions at which resonance phenomena have no time to develope. Some negligible variations of free oscillation amplitudies lead to invariant relations between the frequencies:

$$
Q_{z}^{2}+Q_{r}^{2}=\text { const. }
$$

closed orbit expansion is accompanied by 
the phase shift of the beam centre which is described by the following equation

$$
\frac{d b}{d t}=2-\left(\frac{E_{0}^{2}}{t^{2}}-\eta\right) \frac{e t \cos t}{\beta^{2} E} \text {. }
$$

where e $v \cos \phi$ is the energy gain of the

$w$-th revolution, $E$ is the full energy. The rapid change of the orbit expansion coefficient ( $F i g$. I) makes it necessary to consider the dynamic process since the calculation by formulas (4) and (6) is impeded by the discontinuities of the function $\eta$.

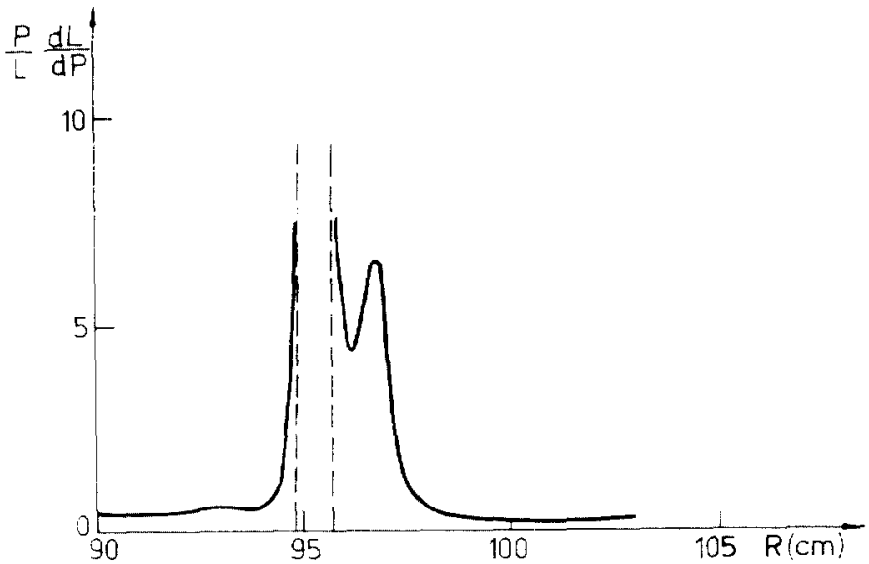

Fig. 1. Jependence of the closed orbit expansion coefficient upon radius.

Dynamical calculations have been performed when the parameters were optimized by numerical calculations of the system of equations in a cylindrical system of coordinates $(r, \phi, z)$ : $r^{\prime \prime}-\frac{2 r^{\prime 2}}{r}-r=-\frac{c}{y}\left(1+\frac{r^{\prime 2}}{r^{2}}+\frac{z^{\prime 2}}{r^{2}}\right)^{12 z}\left\lfloor\left(r^{2}+r^{2}\right) B_{z}-r^{\prime}{ }^{\prime} B_{r}-r^{\prime} B_{b}\right\rfloor-$

$$
-\frac{e t c^{2} r}{p+c}\left(1-\frac{r^{2}}{r^{2}}+\frac{p^{\prime 2}}{r^{2}}\right)
$$

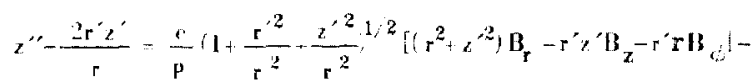

$$
-\frac{1 \cdot x^{2}}{\beta r p}\left(1-\frac{r^{2}}{r^{2}} \frac{z^{2}}{r^{2}}\right)
$$

where is the azimuthal component of the electrical fielis of the accelerating electrode r. 2 long.

A typical picture of particle oscillation in the $r$ and $z$ planes for two last revolutions in the expansion region is shown in Fig. 2 .

Fig. 2 clearly shows the monotonous displacement of the closed orbit which is seen at each period of the magnetic field structure and results in calculated separation of $2-4 \mathrm{~cm}$ per revolution with respect to the chosen observation azimuth.

\section{Shaping the Magnetic Field}

The required dependence of the amplitude and the phase of the th magnetic field harmonic was formed by means of an adultional harmonic coil. The coil is a system of two separate combutors symmet ic with respect to the median plane. They are located along the
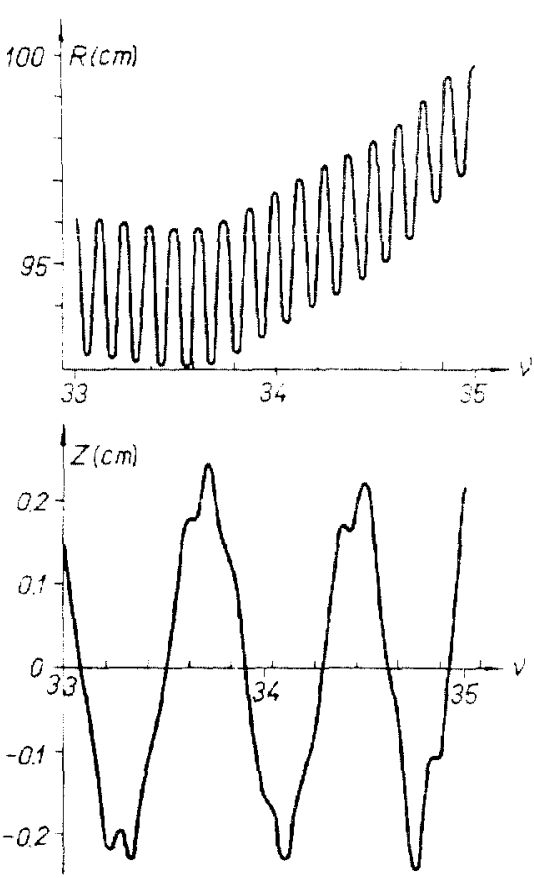

Fig. 2. Effect of closed orbit expansion

radial lines and circumference arcs. for the complete compensation of the mean magnetic field formed with an additional harmonic coil each coil pole is made of two identical branches shifted by a half-period of the system (22. $\left.5^{\circ}\right)$ with currents of opposite directions. With removed chamber covers one can seo the coils and the mechanism for displacing blut Permalloy probe of the measuring device in the radial and azimuthal directions (Fis. 3).

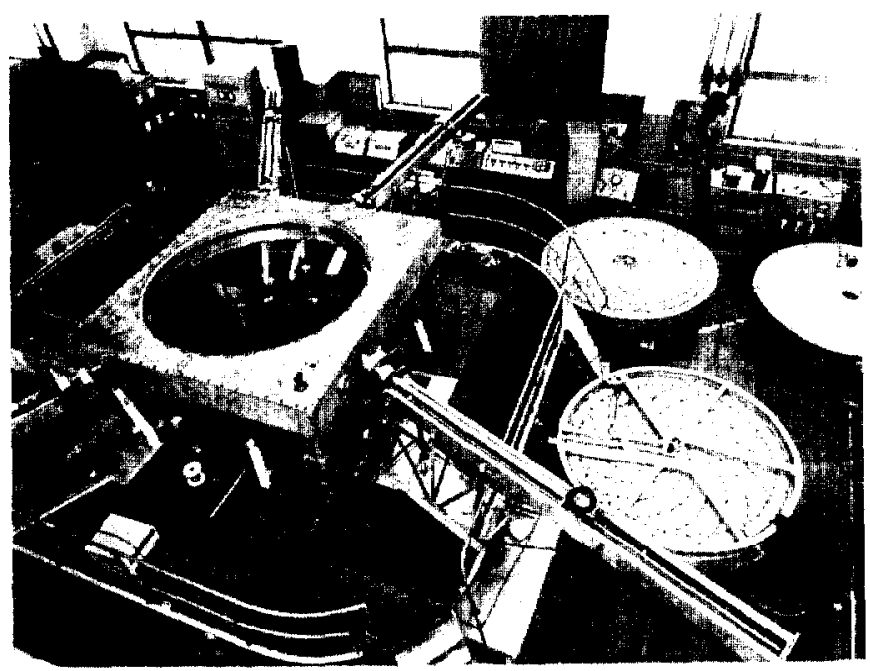

Fig. 3. A general view of the electron nodel during the assembly of additional coils.

Fig. I shows the configuation of the adtitional variation coil remoted rum the meitan plane by 7.05 on. The required lat for whe

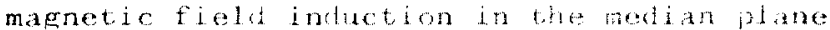


of the orbit expansion region was set by the following expression

$$
\begin{aligned}
& B_{z}=B(r)+\Sigma B_{N}(r) \cos Y[C-6(r) \mid, Y-8,24 \\
& \text { where } \varphi(r)-r \frac{d c}{d r} \max
\end{aligned}
$$

of the rield structure. The limit was imposed only for two harmonics in the structure $\mathrm{N}=8$, $N=24$. The average value of the magnetic field induction along the radius (B(r)) corresponded to the isochronism conditions of the closed not expanded orbits. The tolerance for the amplitude of the magnetic field variation did not exceed a few per cent. There were no special requirements for lower harmonics of the magnetic field structure. As a result of the numerical modelling of the expansion effect an additional condition for the phase of the basic harmonic in the expansion region is found: $\phi \quad=$ const.

The magnetic field distribution for the given radius was measured with 144 equidistant points along the azimuth with a $(0.5+1)$ $\mathrm{cm}$ step. The data of magnetic measurements were recorded on the punched paper tape and then processed by the computer. The accuracy of the magnetic field measurements performert with a Permalloy probe technique is $2 \times 10^{-3}$.

The power supply of the additional harmonic coil has the magnet comparator ${ }^{5}$ which allows one to put the current value with an accuracy better than $10^{-4}$. The power supplies of the other coils have the measuring shunts of the same accuracyb.

The shaped magnetic field in the region of full radii is shown in Fig. 4.

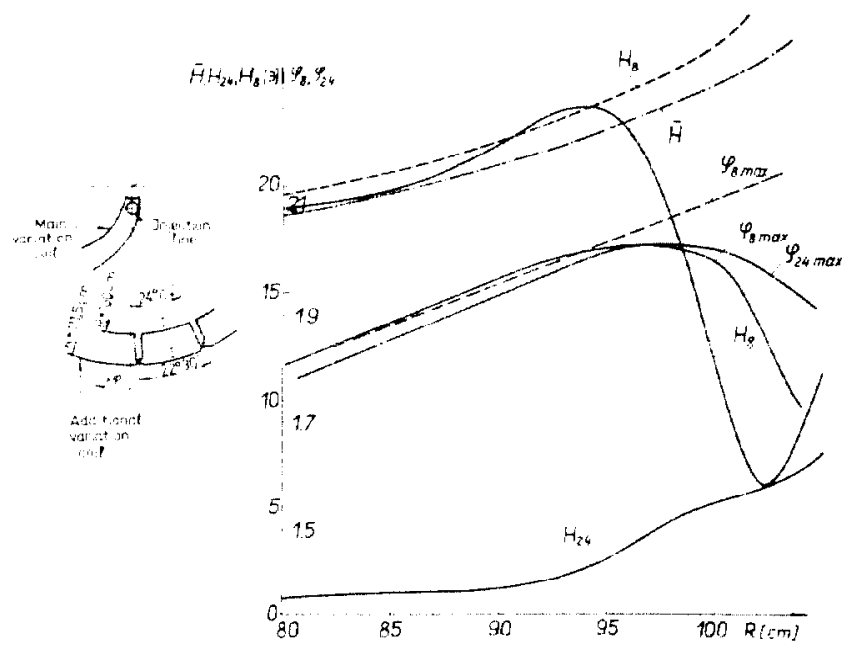

Fig. 4. Basic characteristics of the magnetic field at full radii.

It is seen that the gralient of the th fiel.h harmonic at $R=07.5 \mathrm{~cm}$ is 2.3 Gsicm and corresponds to the required value, and its phase is close to a constant in the radial region $(04-99) \mathrm{cm}$. The magnetic field parameters before the installation of the adilitional soil are shown in the same ficure by dotted lines.

\section{Experiment}

In order to observe experimentally closed orbit expansion five moveable measuring devices were used supplied with differential targets whose collector was projected $2 \mathrm{~mm}$ from the grounded screen.

The computer calculations have shown that with a completely compensated first harmonic of the magnetic field the maximal closed orbit separation could be expected on probes No. 3 and 4 . The separation value should be 4.5 and $4 \mathrm{~cm}$, respectively. The first observations of the expansion effect have confirmed this conclusion. All the probes containing differential targets have proved orbit separation which could be seen in a noticeable current density modulation with respect to radius.

The effect was observed visually by using a fluorescent target mounted on probe xo. 4. When the probe was moved along the radius one could clearly observe a jump of the light spot from the edge of the target to its middle (the fluorescent target was $70 \mathrm{~mm}$ along the radius), which corresponds to the separation of two adjacent orbits of $3.5 \div 4 \mathrm{~cm}$. In order to study the effect in detail a special combined target was mounted on the probe. It is a set of 9 lamelles. The radial size of each of them is $1 \mathrm{~mm}$. The radial size of the last one, mostly remoted from the centre is $18 \mathrm{~mm}$. The lamelles have isolated channels with outputs to a recording device. This target makes it possible to measure, due to proper commutation of channels, the distribution of current density along the radius or the full current.

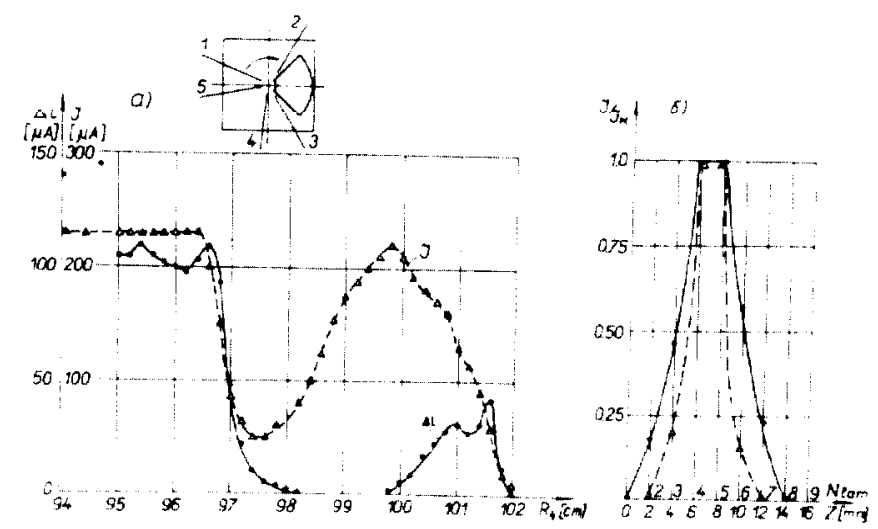

Fig. 5. a) current dependence upon radius ${ }_{i} i$ is the differential target current ( $I R=2 \mathrm{~mm}$ ), $J$ is the integral target current ( $1 \mathrm{H} \ldots 26 \mathrm{~mm})$, b) instant distribution of current density along the vertical. 
Fig. 5a shows two current dependences upon radius measured with the target, i.e., the dependence of the current $d i$ upon radius fixed with lamelles 1 and $2 \quad(\Delta R=2 \mathbf{m m}$ ) and the depentence of the full current $I$ on radius ( $1 \mathrm{R}=26 \mathrm{~mm}$ ). The above figure shows that orbit separation is $4 \mathrm{~cm}$. The completely current-free region at the given azimuth is $2 \mathrm{~cm}$. The current I of a separate orbit $(\mathrm{R}=09.3 \mathrm{~cm}$ ) measured with the integral target equals that before orbit expansion ( $R=04-06.4 \mathrm{~cm}$ ) and is 230 , uA. This result shows the absence of current losses during closed orbit expansion. The integral of the current distribution function of the separated orbit measured with a differential target

( $\left.f \frac{A_{i}}{\lambda_{r}} d r\right)$ is $235, u A$. This proves a good ex-

perimental accuracy. A barrier of $3 \mathrm{~mm}$ radial size was installed in the completely currentfree region $R=08.1-99.8 \mathrm{~cm}$. The displacement of the barrier (within this region) did not affect the value of the full current at $R=99.9 \mathrm{~cm}$ ).

Fig. 5b shows the instant distribution of particle density along the vertical axis obtained with a multi-lamelle target. Fig. 5b shows also that the expansion is accompanied by some increase of the vertical beam size. Thus, before expansion $\left(R_{4}=95 \mathrm{~cm}\right)$ the full width at the distribution basis is $10 \mathrm{~mm}$, while FWHM is $4 \mathrm{~mm}$. After the closed orbit expansion ( $R=100 \mathrm{~cm})$ the full width at the distribution 4 basis is increased to $14 \mathrm{~mm}$, FWHM is $6 \mathrm{~mm}$.

The effect of the closed orbit expansion causes the changes of the phase of the bunch centre of the accelerated beam.

As follows from the dynamical calculations, the beam phase shift is $35-40^{\circ}$ in the narrow radial region of $95-100 \mathrm{~cm}$. The phase of the bunch centre was measured with the apparatus which allows one to follow the phase variation with an accuracy not worse than $2^{\circ}$, the accuracy of its absolute value determination being $+4^{\circ}$. Fig. 6 shows the phase dependence upon radius from which it is seen that at $\$ 5-95 \mathrm{~cm}$ radii the phase of the bunch centre becomes constant and is $10-12^{\circ}$ smallcr than the phase corresponding to the maximal energy

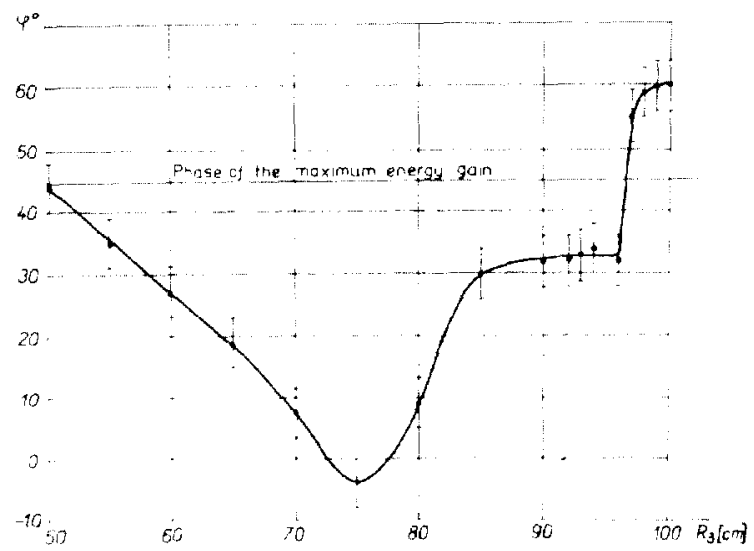

Fig. 6. Dependence of the beam thase upon radius. gain. Then with orbit expansion it jumps $28^{\circ}$. To compensate phase deviation at $60-80 \mathrm{~cm}$ radii no special efforts were made in our experiment. The phase behaviour with respect to radius was confirmed to a good accuracy by the oscillographic measurements. Oscillographic measurements were performed with a Hewlett-Packard 140A sampling oscilloscope an equivalent band of which is $1 \mathrm{GHz}$ with a sensitive vertical deviation of $1 \mathrm{mV} / \mathrm{cm}$, the input resistance being $50 \mathrm{ohm}$. The duration of the accelerated beam pulse was measured by means of the oscilloscope. It was $(4.5+0.2)$ ns at the pulse pedestal and $(2.5+0.2) \mathrm{ns}$ àt half width, which corresponds $\bar{E}_{0}$ the aximuthal bunch width of $65^{\circ}$ and $36^{\circ}$, respectively. The measurements have shown that pulse duration at orbit expansion does not vary within $+0.2 \mathrm{~ns}$ accuracy $\left(+3^{\circ}\right)$.

The beam orbit after expansion was detected with five probes. The orbit position agrees satisfactorily with the computer calculations.

\section{Conclusion}

The theoretical and experimental results of studying the closed orbit expansion in space variation magnetic fields show a possibility of a 100\% beam extraction from the sector cyclic accelerators. They are as follows :

a) middle energy cyclotron (below $1 \mathrm{GeV}$ ) having weak or strong focusing magnetic field structure,

b) ring phasotrons (below $10 \mathrm{GeV}$ ) having a periodic magnetic field 8 structure.

The closed orbit expansion cancells out a very important limitation essential for cyclic accelerators of continuous operation due to the requirement of a great energy gain per revolution which is specific for accelerators of sor type 9. It allows one to solve the problem of particle acceleration using a small number of accelerating cavities in contrast to high current linear accelerators for the same energies 10 .

\section{References}

1. V.P.Dzhelepov, V.P.Dmitrievsky, V.V.Kolga. JINR, P9-7333, Dubna, 1974.

2. V.P.Dmitrievsky, V.V.Kolga, N.I.Polumordvinova. JINR, $P 9-6733$, Dubna, 1972.

3. V.N.Anosov, A.T.Vasilenko, S.B.Vorozhtsov et a1. AE, 25, Ser. 6, 539 (1968).

4. Yu.N.Denisov, A.G.Komissarov, P.T.Shishlyannikov. JJNR, 2983 , Dubna, 1966.

5. Yu.N.Denisov, V.V.Kalinichenko. JINR, 13-6531, Dubna, 1972.

6. Yu.N. Denisov, V.V.KaIinichenko, A.G.Komissarov, Yu.I.Susov, JINR, 13-5068, Dubna, 1970.

7. V.N.Anosov, V.A.Kochkin, D. L. Novikov, L.M. Onischenko. JINR, Po-5873, Dubna, 1971.

S. V.P. Dmitrievsky, V.V.Kolga, N.I. Polumordvinova.Proc. Sth Int.Conf. High Energy Acceler., CERN, 518,1971.

9. F.Russe1. ORNI-34.31, 1064 .

1n. L.Rosen. Proceerlings of the 6th International Conference on High Enerey Accelerators, p.2.37, 1967 . 